Teasing apart socially-induced infertility in non-reproductive female Damaraland molerats, Fukomys damarensis (Rodentia: Bathyergidae)

\title{
A Review Article
}

\begin{abstract}
Nigel C. Bennett
Mammal Research Institute, Department of Zoology and Entomology, University of Pretoria, Pretoria 0002, South Africa.
\end{abstract}

\begin{abstract}
The Damaraland mole-rat is a subterranean mammal exhibiting extreme reproductive skew with a single reproductive female in each colony responsible for procreation. Non-reproductive female colony members are physiologically suppressed while in the colony exhibiting reduced concentrations of plasma luteinizing hormone $(\mathrm{LH})$ and a decreased response of the pituitary, as measured by the release of bioactive $\mathrm{LH}$, to an exogenous dose of gonadotrophin releasing hormone $(\mathrm{GnRH})$. Removal of the reproductive female from the colony results in an elevation of $\mathrm{LH}$ and an enhanced response of the pituitary to a $\mathrm{GnRH}$ challenge in non-reproductive females comparable to reproductive females, implying control of reproduction in these individuals by the reproductive female. The Damaraland mole-rat is an ideal model for investigating the physiological and behavioural mechanisms that regulate the hypothalamopituitary-gonadal axis. In contrast, we know less about the control of reproduction at the level of the hypothalamus. The immunohistochemistry of the GnRH system of both reproductive and non-reproductive female Damaraland mole-rats has revealed no significant differences with respect to morphology, distribution or numbers of immunoreactive GnRH perikarya. We
\end{abstract}


examined whether the endogenous opioid peptide beta-endorphin was responsible for the inhibition of the release of the GnRH from the neurons indirectly by measuring $\mathrm{LH}$ concentrations in these non-reproductive females following single, hourly and eight hourly injections of the opioid antagonist naloxone. The results imply that the endogenous opioid peptide, beta-endorphin, is not responsible for the inhibition of GnRH release from the perikarya in non-reproductive females. Preliminary data examining the circulating levels of cortisol also do not support a role for circulating glucocorticoids. The possible role of kisspeptin is discussed

\section{Introducing the Damaraland mole-rat}

The Damaraland mole-rat (Fukomys damarensis) is a social, subterranean mole-rat which occurs in colonies of around 12 animals (range 2-41) (Bennett and Jarvis, 1988; Jarvis and Bennett, 1993). Damaraland mole-rats are found in semi-arid thorn scrub, woodland savanna and grasslands associated with red Kalahari sands and sandy soils where rainfall is typically low and sporadic (200-400 mm/annum). Burrow temperatures range from a mean of $30^{\circ} \mathrm{C}$ in summer to $19^{\circ} \mathrm{C}$ in winter (Bennett et al. 1988). Endemic to south-western Africa, they have been recorded from NW South Africa, central and N Namibia, SW Zimbabwe, central and N Botswana and extreme W Zambia.

The colony of Damaraland mole-rats comprises a founding reproductive pair and their progeny from several litters; in addition to adults that have migrated into the colony (Burland et al. 2004). The younger offspring of the reproductive animals do not breed while in the natal colony (Bennett and Jarvis, 1988; Bennett et al. 1996). The reproductive pair comprises the most dominant individuals with non-reproductive males being generally more dominant than females (Jacobs et al. 1990). The non-reproductive members of the colony can be placed into work-related groups based on body mass: there is a tendency for smaller (not necessarily 
younger) animals to perform more burrow maintenance than larger (but not necessarily older) animals (Bennett and Jarvis, 1988; Scantlebury et al. 2006).

Reproductively active females exhibit spontaneous ovulation, breed throughout the year and have a gestation of 78-92 days with a post-partum oestrous (Bennett and Jarvis, 1988, Snyman et al. 2006). Mean litter size is 3 (range 1-6, $\mathrm{n}=8$ litters) (Bennett et al.1991).

The oestrus female solicits the male prior to mating and multiple matings occur following a ritualized courtship of tail to tail chasing, vocalizations and head mounting by the sexually excited reproductive female (Bennett and Jarvis, 1988).

Damaraland mole-rats excavate extensive burrow systems that can extend for more than $1 \mathrm{~km}$ and radiate from a central nest and nearby food store (Jarvis et al. 1998). The nest is deep, sometimes in excess of $2.4 \mathrm{~m}$ below the surface, and has two to three entrances. Toilet areas have not been found. Individuals within a colony may remain resident in the same home range for many years ( $>8$ y; N. C. Bennett and J.U.M. Jarvis, unpubl.). The burrow systems are excavated. Mole-rats are herbivorous and excavate numerous superficial foraging tunnels as the animals search for underground storage organs of geophytes (bulbs, tubers and rootstocks). There is selective storage of larger geophytes, although the reason for this selection is unclear: geophytes that are larger may have a longer "shelf life", or it may be more cost effective to transport large geophytes to the store than small ones. Geophytes include various genera of Hyanthaceae (e.g. Dipcadi, Ledebouria, Ornithogalum), Portulaceae (e.g. Talinum) and Cucurbitaceae (e.g. Acanthosicyos), a number of which are toxic to livestock but not to mole-rats. Portable bulbs and corms are stored whereas the very large tubers (e.g. Acanthosicyos) are partly eaten in situ, and will often regenerate (Jarvis et al. 1998; Bennett and Faulkes, 2000). 


\section{Genetic structure of the colonies}

Field and laboratory studies have intimated that the Damaraland mole-rat is an obligate outbreeder and in the past it has been assumed that a colony comprises of an unrelated breeding pair and their natal offspring (Jarvis and Bennett, 1993; Bennett et al. 1996; Rickard and Bennett, 1997). Recent research using the development of microsatellite markers has indeed shown that in wild colonies of the Damaraland mole-rat the breeding pairs are unrelated to one another $(\mathrm{R}=0.02)$. The mean colony relatedness, calculated from relatedness estimates of 15 colonies, is around $\mathrm{R}=0.46$ just less than 0.5 , the expected value for relatedness of first order diploid relatives. These findings clearly demonstrate that normal familial levels of relatedness are sufficient for the evolution of eusociality in this subterranean mammal (Burland et al. 2002).

But what is the nature of paternity in these colonies? Using microsatellite markers Burland et al. (2004) investigated paternity in 18 wild caught colonies of the Damaraland mole-rat to determine whether inbreeding avoidance alone could explain the degree of reproductive skew exhibited. The findings were revolutionary; not only were multiple paternities found within the colonies, but there was also unidentified paternity within colonies. Thus within an individual litter several fathers had contributed their genes to the litter. It was further found that immigrants into the colonies were of both sexes, but predominantly male biased. The findings of these analyses imply that within a wild colony of the Damaraland mole-rat conditions exist where non-reproductive females may come into contact with unrelated or non-kin males, even though they do not disperse from the colony. As a consequence it is inherently apparent that inbreeding avoidance alone would be insufficient for the maintenance of reproductive skew suggesting that the reproductive female somehow suppresses reproduction in these nonreproductive natal females (Bennett et al. 1996). 


\section{Does physiological suppression of reproductive activity occur in non-reproductive}

\section{females?}

For clarity, I use the term repression to describe reproductive inhibition through behavioural means such as incest avoidance, whereas I use the term suppression to describe situations where animals are suppressed physiologically.

In the Damaraland mole-rat, non-reproductive female members that remain philopatric to the colony are often exposed to males which are non-related or unfamiliar kin (Burland et al. 2004). Thus in Damaraland mole-rats incest avoidance alone is not sufficient to ensure that multiple queening does not take place. Unrelated immigrants of both sexes, but mostly males, have the opportunity to enter colonies during windows of opportunity usually following good rains. The rainfall makes the soil friable and promotes soil conditions suitable for burrowing (Burland et a1. 2004; Jarvis and Bennett, 1993). Indeed, DNA fingerprinting studies have revealed that immigrant or floating males not only enter colonies, but can sire offspring and move on (Burland et al. 2004). Unrelated males that either joined the colony and remain or move transiently through the colony are prevented from copulating and reproducing with nonreproductive females as a result of socially-induced physiological suppression. In sum then, inbreeding avoidance alone is insufficient to maintain skew, and multiple reproductive females are prevented by physiological suppression being orchestrated on the non-reproductive females within the natal colony.

In the non-reproductive female Damaraland mole-rat, there appears to be two components to socially induced infertility. Interestingly, both incest avoidance as well as physiological suppression appears to be operational in non-reproductive females. Unlike socially suppressed female naked mole-rats, the ovaries of non-reproductive females are functionally developed possessing primary, secondary, tertiary as well as mature Graafian follicles. However, these females do not possess corpora lutea of ovulation or pregnancy but rather instead possess 
unruptured luteinized follicles which are the result of atresia of the developing follicles (Bennett, 1994; Bennett et al. 1994). In marked contrast, the ovaries of the reproductive female exhibit the normal spectrum of follicular development and corpora lutea (Bennett et al. 1994). If non-reproductive females are removed from the colony and housed on their own or placed in the presence of an unrelated male, the female will undergo ovulation with the subsequent production of corpora lutea of ovulation. Consequently, while they remain in the presence of the colony non-reproductive females remain anovulatory (Molteno and Bennett, 2002).However, non-reproductive females that disperse from the colony may readily undergo ovulation as has been demonstrated by Snyman et al. (2006) who showed spontaneous ovulation can be stimulated to occur if placed in the presence of an unrelated male. A study investigating the concentrations of urinary progesterone in non-reproductive females revealed measurable levels that were significantly higher than those which had been measured in the non-breeding female naked mole-rat, that essentially lack follicular maturation and possess an ovary which for all intents and purposes resembles that of a pre-pubescent female (Kayanja and Jarvis 1971; Faulkes, 1990). These slightly elevated concentrations of progesterone in the non-reproductive female Damaraland mole-rats arise as a result of the luteinisation of unruptured secondary and tertiary follicles (Bennett et al. 1994) (Figure 1). In nonreproductive females removed from the colony and housed singly, these levels rise due to the production of corpora lutea of ovulation (Figure 1).

But what is happening at the level of the pituitary in these non-reproductive females that occur in the colony? It has been demonstrated that non-reproductive female Damaraland mole-rats have reduced levels of circulating bioactive luteinizing hormone (LH). Similarly, nonreproductive female Damaraland mole-rats also exhibit a reduced pituitary responsiveness to exogenous administration of gonadotropin releasing hormone $(\mathrm{GnRH})$ when compared to conspecific reproductive females (Bennett et al. 1993). Rickard and Bennett (1997) demonstrated that if reproductive females were removed from colonies in which all males were 


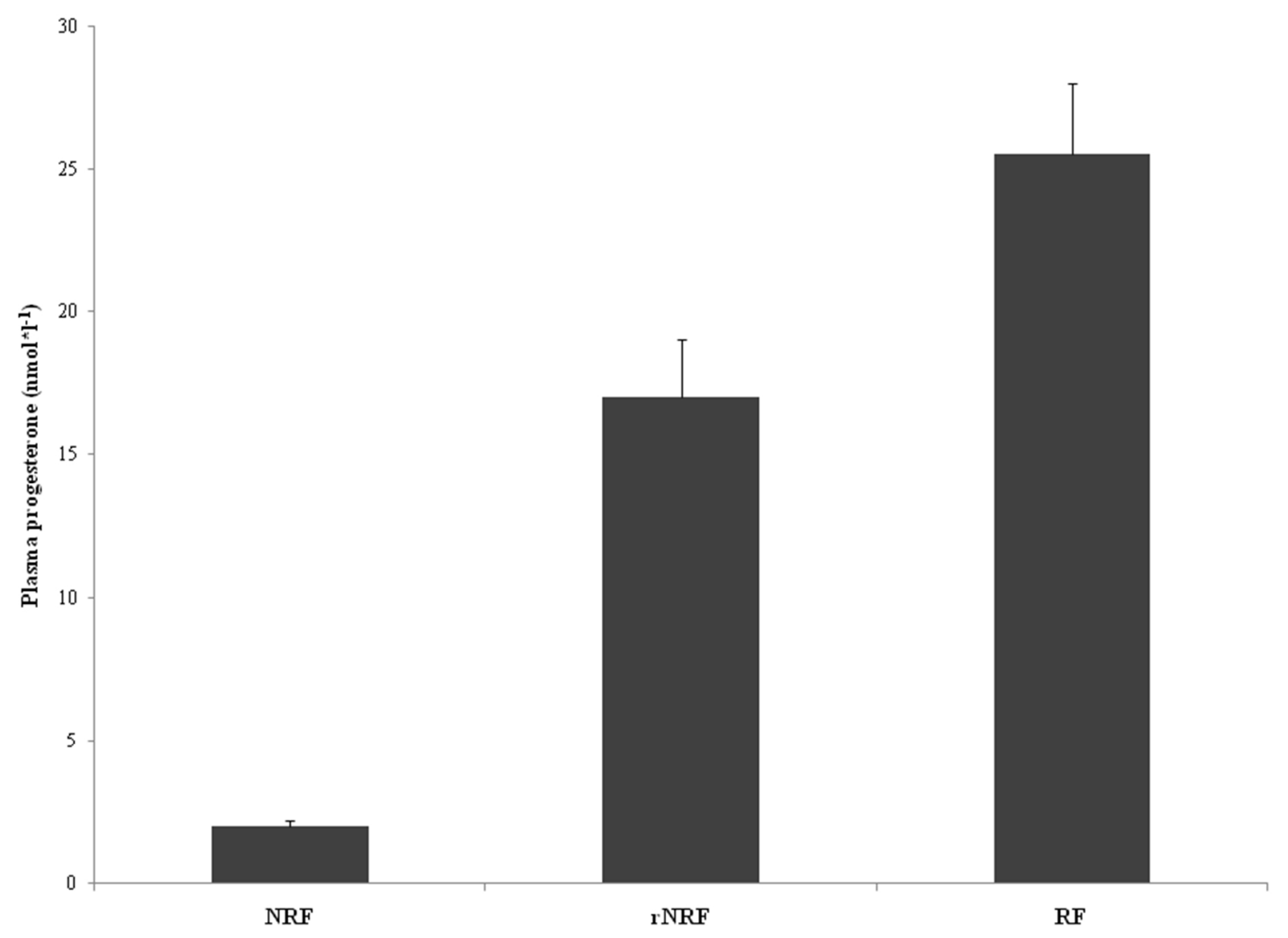

Figure 1 : Mean (and standard error of mean) circulating progesterone concentrations in Damaraland mole-rats. NRF, non-reproductive females; rNRF, nonreproductive females removed from the breeding pair; RF, reproductive females that have produced young. $\mathrm{rNRF}$ vs NRF $P<0.005$, and $\mathrm{RF}$ vNRF<0.0005 (Mann Whitney U-test; from Molteno, 1999). 
related to the breeding pair none of the non-reproductive females would succeed the removed queen to attain breeding status. In stark contrast, in the naked mole-rat, removal of the queen is met by queen succession from a queued non-reproductive female, usually an original litter mate of the queen (Jarvis et al. 1991). Contrary to the naked mole-rat, in the Damaraland mole-rat queen succession is not from within and reproductive recrudescence can only be reinstated by introducing an unrelated adult male to the queenless colony (Rickard and Bennett, 1997).

Indeed, Bennett et al. (1996) revealed that in colonies with queen removal where all offspring are of the natal pair, despite the females showing an elevation of baseline LH (resembling that of a breeding female) and also possessing a similar LH response to the administration of exogenous GnRH as that of the former queen, these same females failed to mate. This provides strong evidence that two components to socially induced infertility are operational (Figure 2).

Within field monitored colonies of the Damaraland mole-rat, a new reproductive female does not come from within the colony, but rather the colony fragments following good rainfall and the colony members pair up with opposite sexed, unrelated and unfamiliar kin to establish new colonies (Bennett et al. 1999). Field and laboratory studies have shown that amongst the diverse members of the genus Fukomys following the death or experimental removal of the reproductive female, breeding ceases until a non related male is placed in the colony (Bennett et al. 1996; Rickard \& Bennett, 1997, Herbst and Bennett, 2001).

In a closely related species of Fukomys, the Mashona mole-rat, Fukomys darlingi, incest avoidance alone appears to be sufficient for social repression of reproduction (Bennett et al. 1997). The Mashona mole-rat occurs in small colonies, 2 to nine individuals and inhabits burrow systems in a mesic environment. However, unlike the Damaraland mole-rat, where non-reproductive females are physiologically suppressed at the level of the hypothalamus (Bennett et al. 1996, Molteno and Bennett 2004), in this species non-reproductive female 


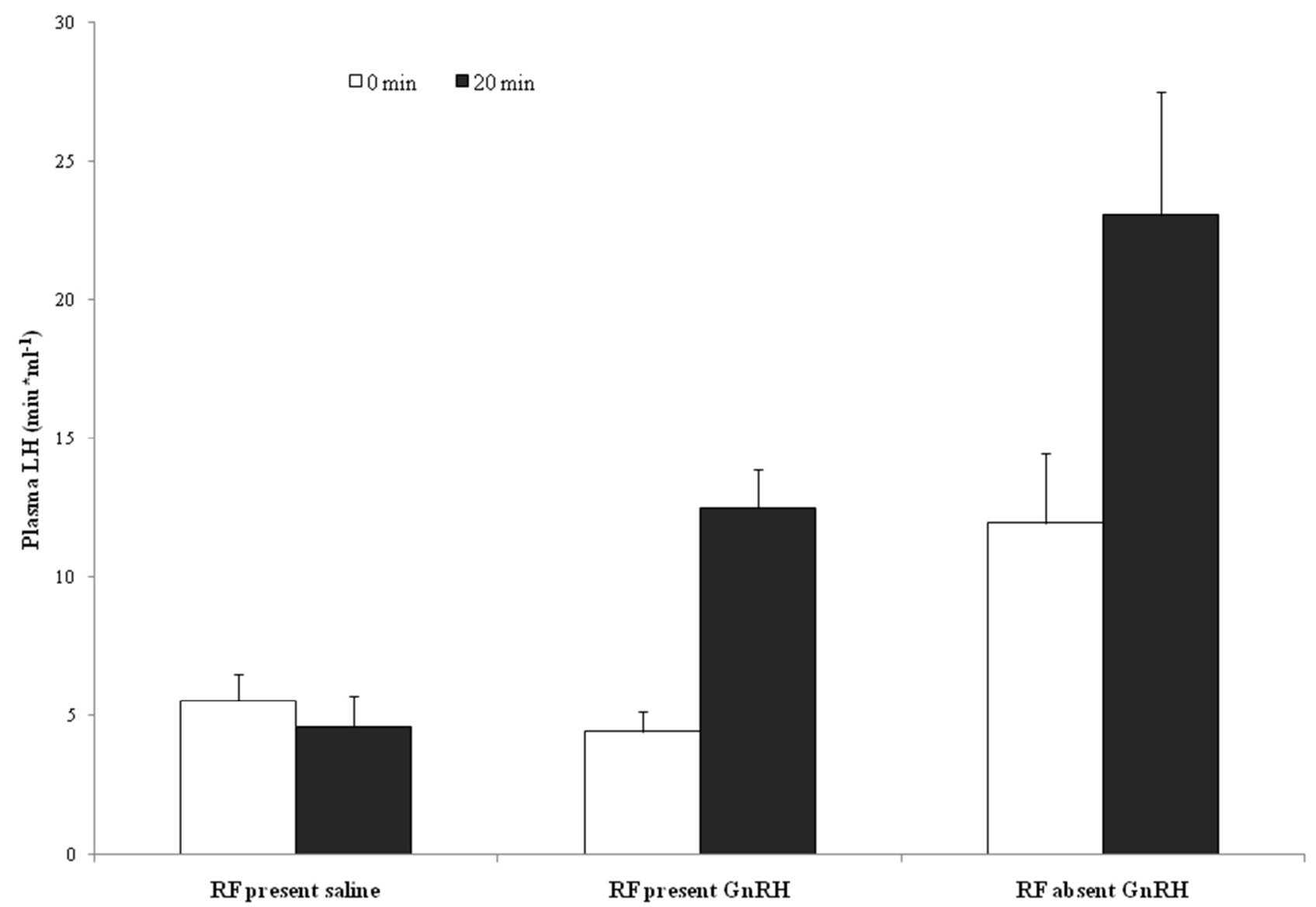

Figure 2 : Concentrations of bioactive plasma LH mean and standard deviation in intact non-reproductive female $F$. damarensis before (0) and 20 min $(20)$ a single injection of saline; a single injection of $2 \mathrm{ug} \mathrm{GnRH}$ in $200 \mathrm{ul}$ physiological saline in non-reproductive females in the presence of the queen; a single $2 \mathrm{ug}$ GnRH in 200ul physiological saline in non-reproductive females in the absence of a queen for 3 years (modified from Bennett et al. 1996). 
members appear to be repressed as a consequence of incest avoidance (Bennett et al. 1997). Indeed, the circulating concentrations of basal LH and the response of the pituitary to an exogenous GnRH challenge are identical between the two groups of females.

These findings pose the question at what level are non-reproductive female Damaraland molerats physiologically suppressed?. In the Damaraland mole-rat, it would appear that the block to ovulation lies at the level of the GnRH secreting neurons. So do reproductive female Damaraland mole-rats possess more GnRH neurons than non-reproductive female conspecifics? A detailed immunohistochemical study has revealed that there are no differences in either numbers of GnRH secreting cells, their morphology or the size of the cell bodies of the GnRH neurons between the reproductive and non-reproductive female Damaraland molerats (Molteno et al. 2004) (Figure 3 ).

Measurements of total pituitary LH revealed that the luteotrophs of the reproductive females were indeed producing more LH than the non-reproductive females (Molteno, 1999) (Figure 4). However, there is a significant difference in the amount of GnRH present in the neurosecretory cells of the two groups (Molteno et al. 2004). Gonadotropin releasing hormone concentrations in the median eminence and proximal pituitary stalk were greater in the non-reproductive females compared to the reproductive females (Figure 5). These findings intimate that the release of GnRH must be inhibited in non-reproductive females resulting in an accumulation of the peptide in the GnRH cells, which leads to an increase in GnRH concentrations in the hypothalami of the non-reproductive females. Interestingly, a comparison of reproductive male and non-reproductive male hypothalamic GnRH revealed no difference, but this is to be expected since there is no physiological suppression of non-reproductive male Damaraland mole-rats (Molteno et al. 2004).

How are the GnRH neurons in the hypothalamus of the non-reproductive females inhibited from releasing the GnRH which is stored in the cell bodies and dendrites? Endogenous opioid peptides are well known to modulate GnRH secretions in many mammalian species (Cicero et 


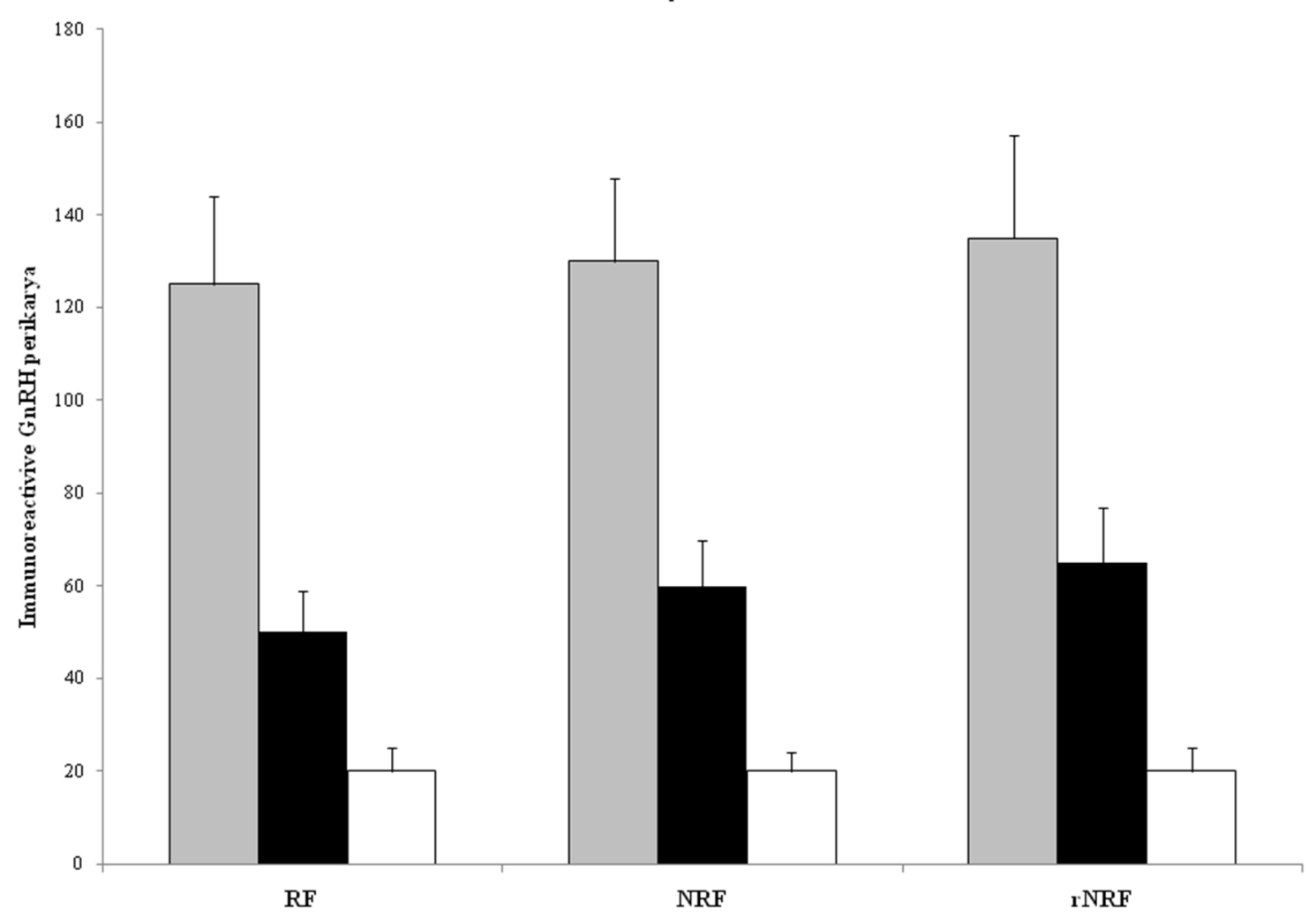

Figure 3 : Mean and standard error of mean number of GnRH immunoreactive neurons detected in every fifth section taken through the forebrain from the confluence of the two hemispheres rostrally, to the posterior hypothalamus caudally through the preoptic area or through the medial basal hypothalamus. RF, Reproductive female; NRF, non-reproductive females; rNRF, non-reproductive females removed from the breeding pair. Mean number of GnRH-ir cell bodies in the whole brain $\mathrm{H}_{2}=0.12 \mathrm{P}>0.05$; the pre-optic area $\mathrm{H}_{2}=0.27, \mathrm{P}>0.05$ and in the medial basal hypothalamus $\mathrm{H}_{2}=0.56 \mathrm{P}>0.05$ ). (modified from Molteno et al. 2004). 


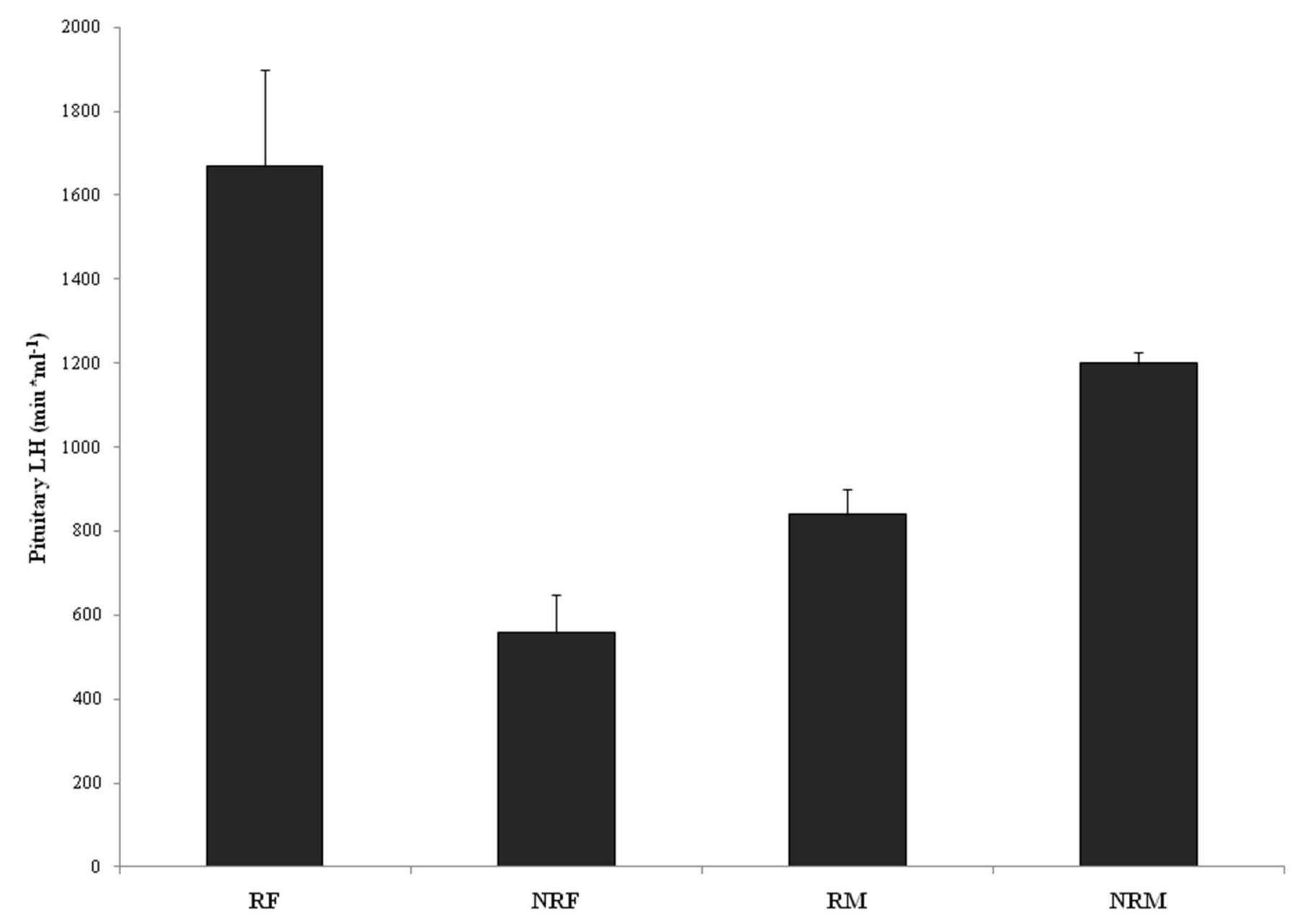

Figure 4 : Concentrations (mean and standard error of mean) of pituitary LH. RF, reproductive females; NRF, non-reproductive females; RM, reproductive males, NRM, non-reproductive males RF vs NRF $\mathrm{P}<0.0002$ and RM vs NRM $\mathrm{P}<0.001$ (Mann Whitney U-test; Molteno, 1999). 


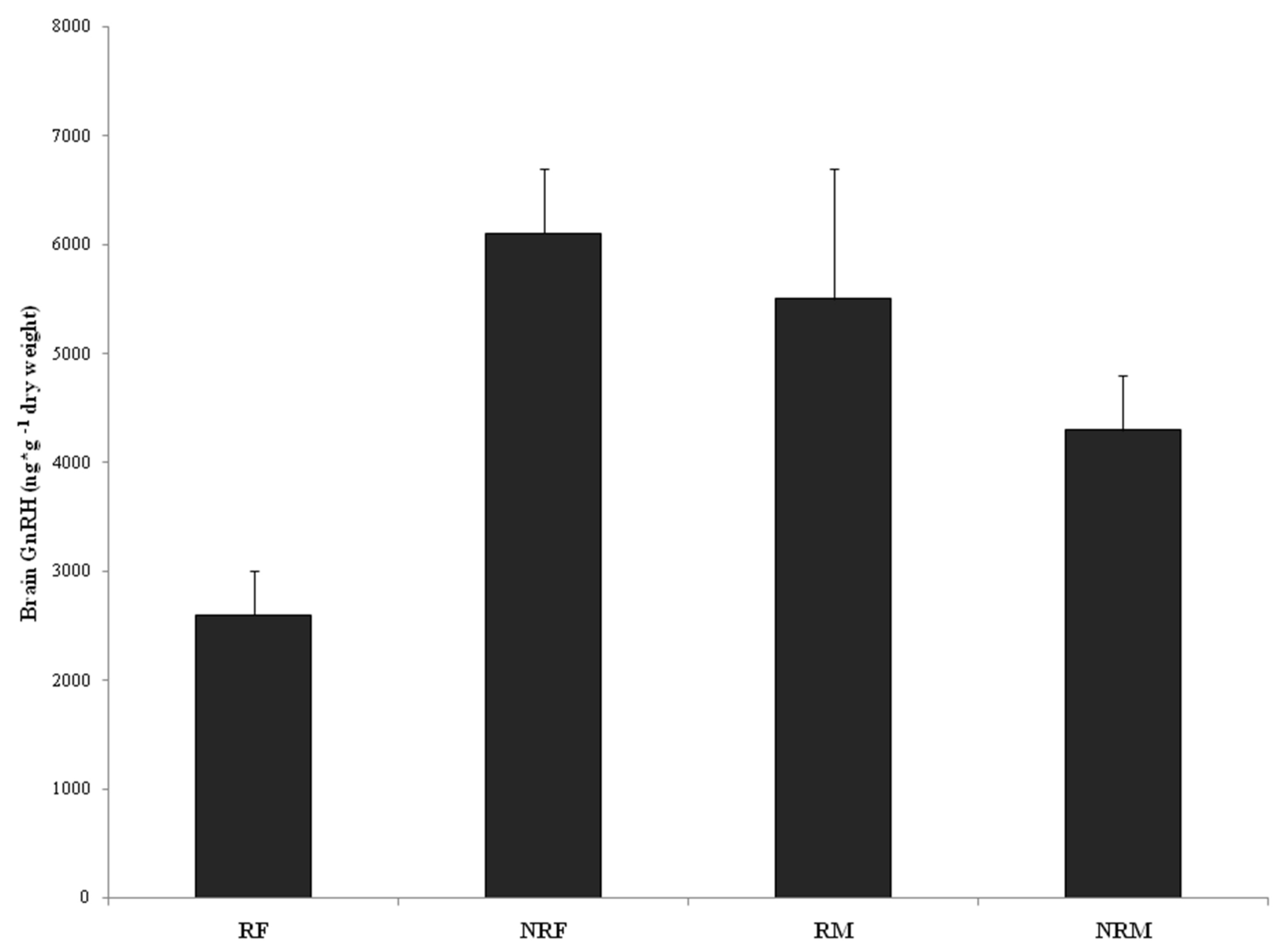

Figure 5 : Concentrations (mean and standard error of mean) of GnRH in whole brains. RF, reproductive females; NRF, non-reproductive females, RM, reproductive male; NRM, non-reproductive males. RF vs NRF P<0.005 (Mann Whitney U-test;. Molteno 1999.) 
al. 1977; Weisner et al. 1984). Indeed, socially induced inhibition of GnRH or LH through increased endogenous opioid peptide activity is a potential neuroendocrine pathway by which infertility through anovulation could be engineered. Endogenous opioid peptides have been found to negatively affect the secretion of gonadotrophin (Almeida 1993). The hypothalamus and pituitary are the two most important sites where opioid peptides may exert their actions upon GnRH and LH secretion. Naloxone, an opioid antagonist was administered to nonreproductive females in an attempt to relieve the hypothalamo-pituitary axis from the potential inhibitory action of endogenous opiod peptides. In a nutshell, Molteno and Bennett (2002) demonstrated that neither a single injection nor multiple injections of naloxone affected basal LH secretion in intact or ovariectomised non-reproductive females implying that sociallyinduced infertility is highly unlikely to be mediated through the endogenous opioid peptide pathway in a steroid dependent or non-dependent way..

Within colonies there is little or no direct aggression between the reproductive and nonreproductive female colony members when in family groups, and this is borne out by the fact that circulating plasma cortisol concentrations measured in ten reproductive females and ten non-reproductive females measured at two distinct time slots $08 \mathrm{~h} 30-09 \mathrm{~h} 00$ and again at $16 \mathrm{~h} 00$ $-16 \mathrm{~h} 30$ were not significantly different between the two groups of females or either time period (Molteno, 1999). These findings imply that non-reproductive females are no more stressed than their reproductive counterparts, intimating that cortisol plays little or no part in the suppression of reproduction in this species.

\section{Possible role of the peptide kisspeptin?}

The recent discovery that a hormone called kisspeptin plays a major role in a number of reproductive functions has opened up a whole new avenue of research for investigating the control of reproduction in socially-suppressed mammals. Kisspeptin is a product of the Kiss 1 gene and forms a ligand to the G protein-coupled receptor, GPR54 (Kotani et al., 2001; Ohtaki et al., 2001). Kisspeptin regulates GnRH secretion and thus forms yet another 
component to the hypothalamo-pituitary-gonadal axis. The GPR54 receptors are co-localised with GnRH neurons in the hypothalamus and may directly stimulate the release of GnRH acting through GPR54 (Messager et al. 2005). In rodents (rats and mice), kisspeptin expression by immunohistochemistry is most noticeable in the arcuate nucleus and the anteroventral periventricular nucleus (AVPV) of the hypothalamus (Smith et al. 2005; Desroziers et al. 2010). Removal of non-reproductive female from a colony (and hence the queen) may bring on the sudden release of kisspeptin and activate ovulation in these females. It is possible that in non-reproductive female Damaraland mole-rats a reduction in the manufacture and release of kisspeptin in the AVPV may in turn inhibit the manufacture and subsequent release of GnRH in these females as has been intimated for the naked mole-rat. This avenue of research is currently being explored (Zhou et al., 2010).

\section{How does reproductive inhibition in non-reproductive females arise?}

Abbott (1988), working on marmosets, and Faulkes (1990), on naked mole-rats, have demonstrated that suppression of reproduction in non-breeding animals in these two social species is orchestrated by overt aggression between the dominant reproductive female and the subordinate non-reproductive females. It is postulated that suppression of reproduction in the non-reproductive females is brought about through stress induced by the dominant and is likely to be controlled by pathways involving endogenous opioid peptides inhibiting GnRH and consequently LH manufacture and release.

Interestingly, in the Damaraland mole-rat the significant difference is that there is a general lack of overt aggression directed by the reproductive female towards either the nonreproductive females or males (Cooney and Bennett, 2000). As a consequence we predicted that the role of endogenous opioid peptides would be minimal and that a different mechanism of repression may be operational (Molteno \& Bennett, 2002). Non-reproductive females have comparable circulating cortisol concentrations intimating that this pathway per se is not responsible for social suppression in the Damaraland mole-rat. Similarly, there is little evidence to implicate the endogenous opioid peptides in the suppression of reproduction in the nonreproductive females. 
Two models have been proposed to explain the lack of reproduction in non-reproductive female colony members, namely the dominant control mechanism and the self restraint mechanism in primate social societies (Snowdon, 1996). Clarke et al. (2001) have suggested that a lack of reproduction in non-reproductive females may actually be due to restraint, where non-reproductive females refrain from ovulating when in the confines of the colony; how this is achieved is still unknown.

\section{Removal of reproductive suppression through the relaxation of ecological constraints}

Molteno \& Bennett (2002) demonstrated that in wild-captured intact colonies the nonreproductive females showed substantially higher pituitary sensitivities to GnRH challenges during periods of high rainfall than when compared the long periods of no rainfall . The rains represent a period when ecological constraints on dispersal are relaxed. These findings intimated that rainfall may well have resulted in the increase in pituitary sensitivity as a result of easing of ecological constraints. Young et al. (2010) following on from this demonstrated that this change in sensitivity of the pituitary during the wet season is not attributed to between female differences, associated change in body mass or concomitant reductions in physiological stress as indicated by circulating urinary cortisol concentrations.

The findings support the notion of reproductive readiness in non-reproductive females during periods of high rainfall when opportunities for dispersal are increased.

\section{Are the non-reproductive males physiologically repressed from reproducing while in the confines of the colony?}

Non-reproductive male Damaraland mole-rats appear not to be physiologically suppressed. Circulating plasma testosterone and urinary testosterone concentrations are indistinguishable between the reproductive groupings (Bennett, 1994). Furthermore, circulating plasma luteinizing hormone (LH) and the pituitary response to exogenous GnRH challenges are similar 
(Bennett et al. 1993). Reproductive males characteristically possess greater testes mass relative to their body mass when compared with non-reproductive male colony members. Interestingly though, this larger testis mass does not result in an increased number of spermatozoa in reproductive males versus non-reproductive males (Faulkes et al. 1994). Non- reproductive males have functional gonads but are oligospermic and occasionally azoospermic, despite comparable sperm numbers (Maswanganye et al. 1999). Interestingly, the follicle stimulating hormone (FSH) levels do not differ between male Damaraland mole-rats irrespective of their reproductive status (Nice et al. 2010). The Damaraland mole-rat therefore conforms more to the usual pattern observed in a socially suppressed male mammal in that there is no obvious physiological suppression and the failure to breed in non-reproductive males apparently results from an exclusion of mating opportunities (Bennett, 1994; Bennett et al. 1996). Thus inbreeding avoidance is properly operational since most of the males within the colony are the offspring of the reproductive pair and all females are related (Jarvis and Bennett, 1993; Burland et al. 1992).

\section{Conclusion}

The studies on Damaraland mole-rats highlight the importance of establishing kin structure and degree of relatedness in wild-captured colonies when trying to unravel the mating system of a social mammal. In a simple family group living in an environment with high ecological constraints on dispersal, the maintenance of reproductive skew may be the result of a lack of unrelated mates for non-reproductive group members to mate with and as a consequence there is no requirement for physiological suppression. However, in mixed kin groups where dispersal is not a common occurrence and infiltration by non-kin conspecifics may arise, a need for physiological repression or dominant control is necessary. The most classic vertebrate example of this is the naked mole-rat, where the queen repels any challenge to her position by 
maintaining a strict dominance hierarchy and physically shoving the conspecifics in the colony (Jarvis et al. 1991, Reeve, 1992).

Reproductive suppression in the Damaraland mole-rat is due to two components: a suppressive action from the social environment, and incest avoidance in the form of obligatory outbreeding. Removal of a non-reproductive female from the colony releases her suppression and her ovulation arises. In a functional colony, the oldest non-reproductive animals are the primary dispersers; however, when either a reproductive male or female dies, the entire colony will fragment. In either instance, dispersal occurs after rainfall when the costs of digging are lowest (Bennett et al. 1996). In this review I have attempted to demonstrate that in order to unravel potential mechanisms of reproductive suppression within social groups, a working knowledge of the degree of relatedness and the measure of multiple paternity operational in the colony is required.

\section{References}

Abbott, DH (1988). Natural suppression of fertility. Symp. Zoological Society of London 60, 7-28.

Almeida, OFX. (1993). Opioids and the neuroendocrine control of reproduction . In A Herz (Ed). Opioids II. Chapt 50. Springer Verlag, Berlin.

Bennett, NC (1988). The trend towards sociality in three species of southern African mole-rats (Bathyergidae): causes and consequences. Unpubl. PhD thesis, University of Cape Town, South Africa.

Bennett NC, Jarvis, JUM (1988). The social structure and reproductive biology of colonies of the mole-rat Cryptomys damarensis (Rodentia Bathyergidae). J. Mammalogy 69, 293302.

Bennett NC, Jarvis, JUM, Davies KC (1988). Daily and seasonal temperatures in the burrows of African rodent moles. South African Journal of Zoology 23, 189-195. 
Bennett NC (1990). Behaviour and social organization in a colony of the Damaraland molerat, Cryptomys damarensis. Journal of Zoology London. 220, 225-248.

Bennett NC, Jarvis JUM, Aguilar GH, McDaid EJ (1991). Growth rates and development in six species of African mole-rats (Family: Bathyergidae). Journal of Zoology London 225, $13-26$.

Bennett NC (1994). Reproductive suppression in social Cryptomys damarensis colonies - a lifetime of socially induced sterility in males and females (Rodentia: Bathyergidae). Journal of Zoology London 234: 25-39.

Bennett NC, Jarvis JUM, Wallace DB 1990. The relative age structure and body masses of complete wild-captured colonies of two social mole-rats, the common mole-rat, Cryptomys hottentotus hottentotus and the Damaraland mole-rat Cryptomys damarensis. Journal of Zoology London. 220, 469-485.

Bennett NC, Jarvis JUM, Millar RP, Sasano H, Ntshinga KV (1994). Reproductive suppression in eusocial Cryptomys damarensis colonies: socially-induced infertility in females. Journal of Zoology London. 233, 617-630.

Bennett NC, Jarvis JUM, Faulkes CG, Millar RP 1993. L.H. responses to single doses of exogenous GnRH by freshly captured Damaraland mole-rats, Cryptomys damarensis. Journal of Reproduction and Fertility. 99,: 81-86

Bennett NC, Faulkes CG, Molteno AJ 1996. Reproductive suppression in subordinate, nonbreeding female Damaraland mole-rats: two components to a lifetime of socially-induced infertility. Proceedings of the Royal Society London Series B. 263, 1599-1603.

Bennett NC, Faulkes CG, Spinks AC (1997). LH responses to single doses of exogenous GnRH by social Mashona mole-rats: a continuum of socially-induced infertility in the family Bathyergidae. Proceedings of the Royal Society London Series B. 264, 10011006. 
Bennett NC, Faulkes CG, Jarvis JUM (1999). Socially-induced infertility, incest avoidance and the monopoly of reproduction in cooperatively breeding African mole-rats. Advances in the Study of Behavior. 28, 75-113.

Bennett NC, Faulkes CG (2000). African mole-rats ecology and eusociality. Cambridge University Press. Cambridge 273pp.

Burland TM, Bennett NC, Jarvis JUM, Faulkes CG (2002). Eusociality in African mole-rats: new insights from patterns of genetic relatedness in the Damaraland mole-rat. Proceedings of the Royal Society London Series B. 269, 1025-1030.

Burland TM, Bennett NC, Jarvis JUM, Faulkes CG (2004). Colony structure and parentage in wild colonies of cooperatively breeding Damaraland mole-rats suggest incest avoidance alone may not maintain reproductive skew. Molecular Ecology 13, 2371-2379.

Cicero TJ, Badger TM, Wilcox CE, Bell RB, Meyer ER (1977). Morphine decreases luteinizing hormone by an action on the hypothalamic-pituitary axis. Journal of Pharmacological Experimental Therapeutics 203, 548-555.

Clarke FM, Miethe GH, Bennett NC (2001). Reproductive suppression in female Damaraland mole-rats Cryptomys damarensis: dominant control or self restraint? Proceedings of the Royal Society London Series B 268, 899-909.

Cooney R M Bennett NC (2000). Incest avoidance and reproductive skew in a cooperatively breeding mammal. Proceedings of the Royal Society London Series B 267, 801-806.

Desroziers E, Mikkelsen J, Simonneaux V, Keller M, Tillet Y, Caraty A. \& Franceschini I. (2010). Mapping of kisspeptin fibres in the brain of the pro-oestrous rat. Journal of Neuroendocrinology 22, 1101-1112

Faulkes CG (1990). Social suppression of reproduction in the naked mole-rat, Heterocephalus glaber. Unpublished $\mathrm{PhD}$ thesis, Univ. of London.

Faulkes CG, Trowell, SN, Jarvis, JUM, Bennett NC(1994). Investigation of sperm numbers and motility in reproductively active and socially suppressed males of two eusocial 
African mole-rats, the naked mole-rat (Heterocephalus glaber) and the Damaraland mole-rat (Cryptomys damarensis). Journal of Reproduction and Fertility. 100, 411-416.

Faulkes CG, Bennett NC, Bruford MW, O’Brien HP, Aguilar GH, Jarvis JUM 1997.

Ecological constraints drive social evolution in the African mole-rats. Proceedings of the Royal Society London Series. B. 264, 1619-1627.

Herbst M, Bennett NC (2001). Recrudescence of sexual activity in a colony of the Mashona mol-rat (Cryptomys darlingi):- a case of incest avoidance. Journal of Zoology, London 254, 163-175.

Jacobs DS, Bennett NC, Jarvis JUM, Crowe TM (1991). The colony structure and dominance hierarchy of the Damaraland mole-rat, Cryptomys damarensis (Rodentia: Bathyergidae) from Namibia. Journal of Zoology, London. 224, 553-576.

Jarvis JUM, Bennett NC 1993. Eusociality has evolved independently in two genera of bathyergid mole-rats - but occurs in no other subterranean mammal. Behavioural Ecology and Sociobiology 33, 353-360.

Jarvis JUM, Bennett NC Spinks AC 1998. A.C. Food availability and foraging by wild colonies of Damaraland mole-rats (Cryptomys damarensis): implications for sociality Oecologia 113, 290-298.

Jarvis JUM (1991). Reproduction of naked mole-rats. In the biology of the naked mole-rat (eds Sherman, P.W., Jarvis, J.U.M. \& Alexander, R.D.) pp. 384-425. Princeton University Press, Princeton. UK.

Kotani M, Detheux M, Vandenbogaede A, Communi, D, Vanderwinden J-M, le Poul E, Brezillon S, Tyldesley R, Suarez-Huerta N, Vandeput F, Blanpain C, Schiffmann SN, Vassart G, Parmentier M (2001). The metastasis suppressor gene KiSS-1 encodes kisspeptins, the natural ligands of the orphan G-protein-coupled receptor GPR54. The Journal of Biological Chemistry 276 (37), 34631-34636. 
Kayanja FIB, Jarvis JUM (1971). Histological observations on the ovary, oviduct and uterus of the naked mole-rat. Zeitshrift fur Saugetierkunde. 36, 114-121.

Maswanganye KA, Bennett NC, Brinders J, Cooney MR 1999.Oligospermia and azoospermia in non-reproductive male Damaraland mole-rats. Journal of Zoology, London 248, 411418

Messager S, Chatzidaki EE, Ma D, Hendrick AG, Zahn D, Dixon J, Thresher RR, Malinge I, Lomet D, Carlton MBL, Colledge WH, Caraty A, Aparicio SAJR (2005). Kisspeptin directly stimulates gonadotropin-releasing hormone release via $\mathrm{G}$ protein-coupled receptor 54. Proceedings of the National Academy of Sciences 102(5), 1761-1766.

Molteno AJ 1999. Reproductive regulation in female Damaraland mole-rats, Cryptomys damarensis: physiological and neuroendocrine mechanisms. Unpubl. MSc thesis, University of Pretoria. 147pp.

Molteno AJ Bennett NC (2000). Anovulation in non-reproductive female Damaraland molerats (Cryptomys damarensis). Journal of. Reproduction and Ferility. 119, 35-41.

Molteno AJ Bennett NC (2002). Rainfall, dispersal and reproductive inhibition in eusocial Damaraland mole-rats (Cryptomys damarensis). Journal of Zoology, London 256, 445448.

Molteno AJ Bennett NC (2002). No evidence for endogenous opioid peptodes in the suppression of the reprodcutive axis in non-breeding female Damaraland mole-rats, Cryptomys damarensis. Hormones and Behavior 41, 115-125.

Molteno AJ, Kallo I, Bennett NC, King JA Coen CW (2004). A neuroanatomical and neuroendocrinological study into the relation between social status and the GnRH System in cooperatively breeding female Damaraland mole-rats, Cryptomys damarensis. Reproduction 127, 13-21. 
Nice PA, Fleming PA, Bennett NC, Bateman PW, Miller D W (2010). Exposure of non-kin females rapidly affects testicular morphology in non-reproductive male Damaraland mole-rats. Journal of Zoology, London. 282, 84-90.

Ohtaki T, Shintani Y, Honda S, Matsumoto H, Hori A, Kanehashi K, Terao Y, Kumano S, Takatsu Y, Masuda Y, Ishibashi Y, Watanabe T, Asada M, Yamada T, Suenaga M, Kitada C, Usuki S, Kurokawa T, Onda H, Nishimura O, Fujono M (2001). Metastasis suppressor gene KiSS-1 encodes peptide ligand of a G-protein-coupled receptor. Nature 411, 613-617.

Reeve HK (1992). Queen activation of lazy workers in colonies of the eusocial naked mole-rat. Nature 358, 147-149.

Rickard CA, Bennett NC (1997). Recrudescence of sexual activity in a reproductively quiescent colony of the Damaraland mole-rat, by the introduction of a genetically unrelated male - a case of incest avoidance in "queenless" colonies. Journal of Zoology, London. 241, 185-202.

Scantlebury M, Speakman JR, Oosthuizen MK, Roper TJ, Bennett NC (2006). Energetics reveals physiologically distinct castes in a eusocial mammal. Nature 440, 795-797.

Smith JT, Cunningham MJ, Rissman EF, Clifton DK, Steiner RA (2005). Regulation of Kiss1 gene expression in the brain of the female mouse. Endocrinology 146 (9), 3686-3692. Snyman PC, Jackson CR, Bennett NC (2006). Do dispersing female Damaraland mole-rats, Cryptomys damarensis (Rodentia: Bathyergidae). Exhibit spontaneous or induced ovulation? Physiology and Behavior 87, 88-94.

Weisner JB, Koenig I, Krulich L, Moss RL (1984). Site of action of beta- endorphin-induced changes in plasma luteinizing hormone and prolactin in the ovariectomised rat. Life Sciences 34, 1463-1473. 
Young AJ, Oosthuizen MK, Lutermann H, Bennett NC (2010). Physiological suppression eases in Damaraland mole-rat societies when ecological constraints on dispersal are relaxed. Hormones and Behavior. 57, 177-183.

Zhou S, Holmes MM, Forger NG, Goldman, BD, Lovern, MB, Caraty A, Faulkes CG, Coen CW (2010). Release from socially-induced reproductive suppression in eusocial naked mole-rats (Heterocephalus glaber) is marked by increased kisspeptinimmunoreactive cell bodies in the hypothalamic anteroventral periventricular nucleus. Neuroscience Conference, San Diego, U.S.A. 